\title{
Efficient Aqueous-Phase Heck and Suzuki Couplings of Aryl Bromides using Tri(4,6- dimethyl-3-sulfonatophenyl)phosphine Trisodium Salt (TXPTS)
}

\author{
Lucas R. Moore and Kevin H. Shaughnessy* \\ Department of Chemistry and the Center for Green Manufacturing, The University of Alabama, \\ Tuscaloosa, AL 35487-0336, USA
}

General. Ligand syntheses were carried out under nitrogen using a dry box and Schlenk techniques unless noted. Coupling reactions were assembled in a drybox in screw-cap vials with a silicone/Teflon ${ }^{\circledR}$ septum or a round bottom sealed with a rubber septum. $\operatorname{Pd}(\mathrm{OAc})_{2}$ and TPPTS were purchased from Strem. Styrene, sodium acrylate, all boronic acids and aryl halides were purchased from Aldrich and were used without further purification. THF and diethyl ether were freshly distilled from sodium-benzophenone ketyl under nitrogen prior to use. Water (deionized) and acetonitrile were degassed by sparging with nitrogen and/or exposure to vacuum.

Tri(4,6-dimethyl-3-sulfonatophenyl)phosphine (TXPTS). ${ }^{1}$ Fuming sulfuric acid (2.5mL, $4.75 \mathrm{mmol}$ ) was added to a round bottom flask under nitrogen via syringe. The round bottom was placed in a brine/ice bath. Finely ground tri(2,4-dimethylphenyl)phosphine (999.0 mg, 2.89 mmol) was added to the round bottom slowly under nitrogen. The reaction mixture was allowed to warm to room temperature and stir for 3 hours. The reaction flask was placed back in the brine/ice bath and ice $(\sim 10 \mathrm{~g})$ was added to the reaction flask over a 30 minute period. $\mathrm{NaOH}$ solution (3.85 $\mathrm{g}$ in $30 \mathrm{~mL} \mathrm{H} \mathrm{H}_{2} \mathrm{O}$ ) was added to the reaction flask. A saturated $\mathrm{NaOH}$ solution was then added dropwise to reach a $\mathrm{pH}$ of 7 . The resulting mixture was extracted with $\mathrm{MeOH}(100$ $\mathrm{mL})$ and filtered. The remaining solid was washed with a $\mathrm{MeOH} / \mathrm{H}_{2} \mathrm{O}$ solution $(80 \mathrm{ml} \mathrm{MeOH}, 4$ $\mathrm{mL} \mathrm{H}_{2} \mathrm{O}$ ). Removal of solvent from the combined $\mathrm{MeOH}$ filtrates under reduced pressure gave a white solid that was again extracted into $\mathrm{MeOH}(40 \mathrm{~mL})$. The mixture was filtered and the solvent removed from the filtrate to give TXPTS $(1.61 \mathrm{~g}, 85.6 \%)$ as a colorless solid. ${ }^{1} \mathrm{HNMR}$ (360 MHz, CD $\left.\mathrm{CD}_{3} \mathrm{OD}\right) \delta 7.32$ (d, $\left.J=4.93 \mathrm{~Hz}, 3 \mathrm{H}\right), 7.25$ (d, $\left.J=4.93 \mathrm{~Hz}, 3 \mathrm{H}\right), 2.59$ (s, 9H), 2.31 (s, $9 \mathrm{H}) ;$ ) ${ }^{13} \mathrm{C} \mathrm{NMR}\left(90.5 \mathrm{MHz}, \mathrm{CDCl}_{3}\right): \delta 147.5\left(\mathrm{~d}, J_{\mathrm{C}-\mathrm{P}}=26.34 \mathrm{~Hz}\right), 140.5,138.8,135.4\left(\mathrm{~d}, J_{\mathrm{C}-\mathrm{P}}=\right.$ $4.16 \mathrm{~Hz}), 132.5,131.5\left(\mathrm{~d}, J_{\mathrm{C}-\mathrm{P}}=8.33 \mathrm{~Hz}\right), 21.4\left(\mathrm{~d}, J_{\mathrm{C}-\mathrm{P}}=46.61 \mathrm{~Hz}\right), 20.6,{ }^{31} \mathrm{P} \mathrm{NMR}(202.5 \mathrm{MHz}$, $\left.\mathrm{CD}_{3} \mathrm{OD}\right): \delta-30.3$.

Tri(4-methoxy-2-methylphenyl)phosphine. Magnesium turnings (1.1480 g, $47.22 \mathrm{mmol})$ were added to a round bottom equipped with a stirbar under nitrogen. THF (45mL) and 4bromo-3-methylanisole $(9.38 \mathrm{~g}, 46.64 \mathrm{mmol})$ was added to the round bottom via syringe. The reaction mixture was allowed to stir under reflux for several hours. Trichlorophosphine (1.26 $\mathrm{ml}, 14.4 \mathrm{mmol}$ ) was dissolved in THF (40 mL) in a second round bottom flask under $\mathrm{N}_{2}$. The Grignard solution was cooled to $-78^{\circ} \mathrm{C}$, and the phosphine solution was added drop-wise via cannula. The reaction mixture was allowed to warm to room temperature and stir overnight. The solution was quenched with a saturated solution of ammonium chloride $(100 \mathrm{~mL})$. The product was extracted with toluene, dried with $\mathrm{MgSO}_{4}$, and dried under vacuum ( 0.1 torr). The crude material was purified by flash chromatography $\left(\mathrm{SiO}_{2}\right)$ eluting with 1:1 hexane: $\mathrm{CH}_{2} \mathrm{Cl}_{2}$ to give the product as a colorless solid $(4.79 \mathrm{~g}, 76 \%) .{ }^{1} \mathrm{H} \mathrm{NMR}\left(360 \mathrm{MHz}, \mathrm{CDCl}_{3}\right): \delta 6.77(\mathrm{~s}, 1 \mathrm{H})$, $6.3(\mathrm{~m}, 2 \mathrm{H}), 3.79(\mathrm{~s}, 3 \mathrm{H}), 2.36(\mathrm{~s}, 3 \mathrm{H}) \cdot{ }^{13} \mathrm{C} \mathrm{NMR}\left(90.5 \mathrm{MHz}, \mathrm{CDCl}_{3}\right): \delta 160.0,144.1\left(\mathrm{~d}, J_{\mathrm{C}-\mathrm{P}}=\right.$ $27.75 \mathrm{~Hz}), 134.4,126.2\left(\mathrm{~d}, J_{\mathrm{C}-\mathrm{P}}=8.33 \mathrm{~Hz}\right), 115.8\left(\mathrm{~d}, J_{\mathrm{C}-\mathrm{P}}=5.54 \mathrm{~Hz}\right), 111.4,55.0,21.3\left(\mathrm{~d}, J_{\mathrm{C}-\mathrm{P}}=\right.$ 20.81 Hz). ${ }^{31} \mathrm{P}$ NMR (202.5 MHz, $\left.\mathrm{CDCl}_{3}\right): \delta$-34. (s). 
Tri(4-methoxy-6-methyl-3-sulfonatophenyl)phosphine (TMAPTS). Fuming sulfuric acid $(2.5 \mathrm{~mL}, 4.75 \mathrm{mmol})$ was added to a round bottom flask under nitrogen via syringe. The round bottom was placed in a brine/ice bath. Finely ground tri(4-methoxy-2-methylphenyl)phosphine $(1.26 \mathrm{~g}, 3.18 \mathrm{mmol})$ was added to the round bottom slowly under nitrogen. The reaction mixture was allowed to warm to room temperature and stir for 3 hours. The reaction flask was placed back in the brine/ice bath and ice $(\sim 10 \mathrm{~g})$ was added to the reaction flask over a 30 minute period. $\mathrm{NaOH}$ solution $\left(3.85 \mathrm{~g}\right.$ in $30 \mathrm{~mL} \mathrm{H}_{2} \mathrm{O}$ ) was added to the reaction flask. A saturated $\mathrm{NaOH}$ solution was then added dropwise to reach a $\mathrm{pH}$ of 7 . The resulting mixture was extracted with $\mathrm{MeOH}(100 \mathrm{~mL})$ and filtered. The remaining solid was washed with a $\mathrm{MeOH} / \mathrm{H}_{2} \mathrm{O}$ solution ( $80 \mathrm{ml} \mathrm{MeOH}, 4 \mathrm{~mL} \mathrm{H}_{2} \mathrm{O}$ ). Removal of solvent from the combined $\mathrm{MeOH}$ filtrates under reduced pressure gave a white solid that was again extracted into $\mathrm{MeOH}(40 \mathrm{~mL})$. The mixture was filtered and the solvent removed from the filtrate to give TMAPTS $(1.08 \mathrm{~g}, 54 \%)$ as a colorless solid. ${ }^{1} \mathrm{HNMR}\left(360 \mathrm{MHz}, \mathrm{CD}_{3} \mathrm{OD}\right): \delta 6.82(\mathrm{~d}, J=4.32 \mathrm{~Hz}, 3 \mathrm{H}), 6.39(\mathrm{~d}, J=3.69 \mathrm{~Hz}$, $3 \mathrm{H}), 3.31(\mathrm{~s}, 9 \mathrm{H}), 1.75(\mathrm{~s}, 9 \mathrm{H}) .{ }^{13} \mathrm{C} \mathrm{NMR}\left(90.5 \mathrm{MHz}, \mathrm{CD}_{3} \mathrm{OD}\right): \delta 158.9,149.5\left(\mathrm{~d}, J_{\mathrm{C}-\mathrm{P}}=26.36\right.$ $\mathrm{Hz}), 134.6,131.2,125.0\left(\mathrm{~d}, J_{\mathrm{C}-\mathrm{P}}=8.32 \mathrm{~Hz}\right), 115.9\left(\mathrm{~d}, J_{\mathrm{C}-\mathrm{P}}=4.16 \mathrm{~Hz}\right), 57.00,22.0\left(\mathrm{~d}, J_{\mathrm{C}-\mathrm{P}}=20.81 \mathrm{~Hz}\right)$. ${ }^{31} \mathrm{P}$ NMR (202.5 MHz, $\left.\mathrm{CD}_{3} \mathrm{OD}\right): \delta-29.5$ (s).

Representative procedure for the Heck coupling reactions with styrene (Table 1). $\mathrm{Pd}(\mathrm{OAc})_{2}\left(5.6 \mathrm{mg}, 0.025 \mathrm{mmol}\right.$ ), TXPTS (43.7 mg, $0.075 \mathrm{mmol}$ ), and $\mathrm{Na}_{2} \mathrm{CO}_{3}(212 \mathrm{mg}, 2.0$ mmol) were added to a $50 \mathrm{~mL}$ round bottom flask equipped with a stir bar and rubber septum while in the dry box. Upon removing from dry box, aryl halide (1.00 mmol), styrene (156 mg, $1.50 \mathrm{mmol})$, and degassed $1: 1 \mathrm{CH}_{3} \mathrm{CN}: \mathrm{H}_{2} \mathrm{O}(10 \mathrm{~mL})$ were added via syringe. The round bottom was placed in an oil bath at $80{ }^{\circ} \mathrm{C}$, and allowed to stir until complete as determined by GC (2-4 h). The reaction was poured into saturated sodium carbonate $(50 \mathrm{~mL})$, extracted with ethyl acetate $(3 \times 30 \mathrm{~mL})$, and dried with $\mathrm{MgSO}_{4}$. Products were purified by flash chromatography $\left(\mathrm{SiO}_{2}\right)$.

$\boldsymbol{E}$-4-Ethylstilbene (entry 1). Using the general procedure, 1-ethyl-4-iodobenzene (232 mg, $1.00 \mathrm{mmol}$ ) was coupled with styrene. The crude product was chromatographed eluting with hexane: $\mathrm{CH}_{2} \mathrm{Cl}_{2}$ :EtOAc (70:20:10) to give $E$-4-ethylstilbene as a white solid (187.0 $\mathrm{mg}, 90 \%$ yield). $\mathrm{mp} 84-86{ }^{\circ} \mathrm{C}$ (lit. $\left.{ }^{2} \mathrm{mp} 89-90{ }^{\circ} \mathrm{C}\right) .{ }^{1} \mathrm{HNMR}\left(360 \mathrm{MHz}, \mathrm{CDCl}_{3}\right): \delta 7.49(\mathrm{~d}, J=7.40 \mathrm{~Hz}$, $2 \mathrm{H}), 7.43(\mathrm{~d}, J=8.02 \mathrm{~Hz}, 2 \mathrm{H}), 7.34(\mathrm{t}, J=7.39 \mathrm{~Hz}, 2 \mathrm{H}), 7.19(\mathrm{~m}, 3 \mathrm{H}), 7.07(\mathrm{~d}, J=2.47 \mathrm{~Hz}, 2 \mathrm{H})$, 2.65 (q, $\left.J=7.40 \mathrm{~Hz}, 2 \mathrm{H}), 1.25(\mathrm{t}, J=8.01 \mathrm{~Hz}, 3 \mathrm{H}) .{ }^{13} \mathrm{C} \mathrm{NMR} 90.6 \mathrm{MHz}, \mathrm{CDCl}_{3}\right): \delta 144.3$, $137.9,135.2,129.0,128.6,128.5,128.1,127.7,126.9,126.7,29.0,15.9$.

$\boldsymbol{E}$-4-Methylstilbene ${ }^{3}$ (entry 2). Using the general procedure, 4-bromotoluene (173.8 $\mathrm{mg}, 1.00$ mmol) was coupled with styrene. The crude product was chromatographed eluting with hexane: $\mathrm{CH}_{2} \mathrm{Cl}_{2}$ :EtOAc (70:20:10) to give $E$-4-methylstilbene as a white solid (176.6 mg, $90 \%$ yield). mp 112-115 ${ }^{\circ} \mathrm{C}$. ${ }^{1} \mathrm{HNMR}\left(360 \mathrm{MHz}, \mathrm{CDCl}_{3}\right): \delta 7.46(\mathrm{~d}, J=7.40 \mathrm{~Hz}, 2 \mathrm{H}), 7.42$ (d, J=8.02 $\mathrm{Hz}, 2 \mathrm{H}), 7.35(\mathrm{~m}, 2 \mathrm{H}), 7.25(\mathrm{~m}, 1 \mathrm{H}), 7.17(\mathrm{~m}, 2 \mathrm{H}), 7.07(\mathrm{~d}, J=1.85 \mathrm{~Hz}, 2 \mathrm{H}), 2.36(\mathrm{~s}, 3 \mathrm{H}) .{ }^{13} \mathrm{C}$ NMR (90.6 MHz, $\left.\mathrm{CDCl}_{3}\right): \delta 138.1,135.1,130.0,129.4,129.1,129.0,128.3,127.8,127.0$, $126.8,21.0$.

$\boldsymbol{E}$-4-Acetylstilbene ${ }^{4}$ (entry 3). Using the general procedure, 4-bromoacetophenone (214.1 $\mathrm{mg}, 1.00 \mathrm{mmol}$ ) was coupled with styrene. The crude product was chromatographed eluting with hexane: $\mathrm{CH}_{2} \mathrm{Cl}_{2}$ :EtOAc (70:20:10) to give $E$-4-acetylstilbene as a white solid (224.2 mg, 
94\% yield). mp 134- $137{ }^{\circ} \mathrm{C} .{ }^{1} \mathrm{HNMR}\left(360 \mathrm{MHz}, \mathrm{CDCl}_{3}\right): \delta 7.95(\mathrm{~d}, J=8.02,2 \mathrm{H}), 7.58(\mathrm{~d}$, $J=8.02 \mathrm{~Hz}, 2 \mathrm{H}), 7.54(\mathrm{~d}, J=7.4 \mathrm{~Hz}, 2 \mathrm{H}) 7.26(\mathrm{~m}, 5 \mathrm{H}), 2.60(\mathrm{~s}, 3 \mathrm{H}) .{ }^{13} \mathrm{C}$ NMR $(90.6 \mathrm{MHz}$, $\left.\mathrm{CDCl}_{3}\right): \delta 197.8,142.3,137.0,136.3,131.8,129.2,129.1,128.6,127.8,127.1,126.8,26.9$.

$\boldsymbol{E}$-4-Methoxy-2-methylstilbene (entry 4). Using the general procedure, 4-bromo-3methylanisole $(199.4 \mathrm{mg}, 1.00 \mathrm{mmol})$ was coupled with styrene. The crude product was chromatographed eluting with hexane: $\mathrm{CH}_{2} \mathrm{Cl}_{2}$ :EtOAc (70:20:10) to give $E$-4-methoxy-2methylstilbene as a white solid $(172.5 \mathrm{mg}, 78 \%$ yield $) . \mathrm{mp} 67-70{ }^{\circ} \mathrm{C} .{ }^{1} \mathrm{HNMR}(360 \mathrm{MHz}$, $\left.\mathrm{CDCl}_{3}\right): \delta 7.52(\mathrm{~m}, 3 \mathrm{H}), 7.30(\mathrm{~m}, 4 \mathrm{H}), 6.90(\mathrm{~m}, 1 \mathrm{H}), 6.80(\mathrm{~m}, 2 \mathrm{H}), 3.38(\mathrm{~s}, 3 \mathrm{H}), 2.40(\mathrm{~s}, 3 \mathrm{H}) .{ }^{13} \mathrm{C}$ $\operatorname{NMR}\left(90.6 \mathrm{MHz}, \mathrm{CDCl}_{3}\right): \delta 159.4,138.3,137.6,133.1,128.9,128.4,127.5,126.9,126.6$, 126.4, 116.0, 112.0, 54.6, 20.1.

Representative procedure for the Heck coupling reactions with sodium acrylate (Table 1). $\mathrm{Pd}(\mathrm{OAc})_{2}(5.6 \mathrm{mg}, 0.025 \mathrm{mmol})$, TXPTS $(43.7 \mathrm{mg}, 0.075 \mathrm{mmol})$, sodium acrylate $(141 \mathrm{mg}$, $1.50 \mathrm{mmol})$, and $\mathrm{Na}_{2} \mathrm{CO}_{3}(212 \mathrm{mg}, 2.0 \mathrm{mmol})$ were added to a round bottom equipped with a stir bar and rubber septum while in the dry box. Upon removing from dry box, the aryl bromide $(1.00 \mathrm{mmol})$ and degassed $1: 1 \mathrm{CH}_{3} \mathrm{CN}: \mathrm{H}_{2} \mathrm{O}(10 \mathrm{~mL})$ were added via syringe. The round bottom was placed in an oil bath at $80{ }^{\circ} \mathrm{C}$ and allowed to stir for several hours. The reaction mixture was added to saturated sodium carbonate $(50 \mathrm{~mL})$. The residual aryl bromide was extracted with ethyl acetate $(3 \times 30 \mathrm{~mL})$. The $\mathrm{pH}$ of the aqueous phase was then brought to ca. $1 \mathrm{using}$ concentrated $\mathrm{H}_{2} \mathrm{SO}_{4}$. The cinnamic acid product was extracted with $\mathrm{CH}_{2} \mathrm{Cl}_{2}(3 \times 30 \mathrm{~mL})$ and the combined organic extracts were dried over $\mathrm{MgSO}_{4}$. Removal of solvent under reduced pressure gave the crude product, which was recrystallized from $\mathrm{H}_{2} \mathrm{O}$ /Ethanol.

$\boldsymbol{E}$-4-Methylcinnamic acid (entry 5). Using the general procedure, 4-bromotoluene (173.8 $\mathrm{mg}, 1.00 \mathrm{mmol}$ ) was coupled with sodium acrylate. Using the general work-up procedure gave E-4-methylcinnamic acid as a white solid (133.9 mg, 81\% yield). mp 197-199 ${ }^{\circ} \mathrm{C}$ (lit. ${ }^{5} \mathrm{mp} 198$ $\left.200{ }^{\circ} \mathrm{C}\right) .{ }^{1} \mathrm{HNMR}\left(360 \mathrm{MHz}, \mathrm{CDCl}_{3}\right): \delta 10.13$ (brs, $\left.1 \mathrm{H}\right), 7.57(\mathrm{~m}, 3 \mathrm{H}), 7.23(\mathrm{~d}, J=8.01 \mathrm{~Hz}, 2 \mathrm{H})$, $6.47(\mathrm{~d}, J=16.03 \mathrm{~Hz}, 1 \mathrm{H}), 2.32(\mathrm{~s}, 3 \mathrm{H}) .{ }^{13} \mathrm{C} \mathrm{NMR}\left(90.6 \mathrm{MHz}, \mathrm{CDCl}_{3}\right): \delta 168.6,144.8,141.1$, $132.4,130.4,129.1,119.0,21.9$. NMR spectra were identical to published spectra. ${ }^{6}$

$\boldsymbol{E}$-4-Methoxycinnamic acid ${ }^{7}$ (entry 6). Using the general procedure, 4-bromo-3methylanisole $(186.8 \mathrm{mg}, 1.00 \mathrm{mmol}$ ) was coupled with sodium acrylate. Using the general work-up procedure gave $E$-4-methoxycinnamic acid as a white solid (140.6 mg, $79 \%$ yield). mp 173-174 ${ }^{\circ} \mathrm{C}$ (lit. $\left.{ }^{5} \mathrm{mp} 174{ }^{\circ} \mathrm{C}\right) .{ }^{1} \mathrm{HNMR}\left(360 \mathrm{MHz}, \mathrm{CDCl}_{3}\right): \delta 10.22$ (brs, 1H), 7.67 (d, J=8.63 $\mathrm{Hz}, 2 \mathrm{H}), 7.57(\mathrm{~d}, J=16.03 \mathrm{~Hz}, 1 \mathrm{H}), 6.99(\mathrm{~d}, J=8.63 \mathrm{~Hz}, 2 \mathrm{H}), 6.41(\mathrm{~d}, J=16.03 \mathrm{~Hz}, 1 \mathrm{H}), 3.84$ (s, $3 \mathrm{H}) .{ }^{13} \mathrm{C}$ NMR $\left(90.6 \mathrm{MHz}, \mathrm{CDCl}_{3}\right): \delta 168.7,161.9,144.6,130.8,127.8,117.4,115.3,56.2$.

$\boldsymbol{E}$-2-Methylcinnamic acid $^{8}$ (entry 7). Using the general procedure, 2-bromotoluene (170.6 $\mathrm{mg}, 1.00 \mathrm{mmol}$ ) was coupled with sodium acrylate. Using the general work-up procedure gave E-2-methylcinnamic acid as a white solid (156.4 mg, 82\% yield). mp 169-171 ${ }^{\circ} \mathrm{C}$ (lit. mp 175$\left.176{ }^{\circ} \mathrm{C}\right) .{ }^{1} \mathrm{HNMR}\left(360 \mathrm{MHz}, \mathrm{CDCl}_{3}\right): \delta 10.00$ (brs, $\left.1 \mathrm{H}\right), 7.86(\mathrm{~d}, J=15.42 \mathrm{~Hz}, 1 \mathrm{H}), 7.72(\mathrm{~d}$, $, J=7.4 \mathrm{~Hz} 1 \mathrm{H}), 7.30(\mathrm{~m}, 3 \mathrm{H}), 6.45(\mathrm{~d}, J=15.41 \mathrm{~Hz}, 1 \mathrm{H}), 2.42(\mathrm{~s}, 3 \mathrm{H}) .{ }^{13} \mathrm{C} \mathrm{NMR}(90.6 \mathrm{MHz}$, $\left.\mathrm{CDCl}_{3}\right): \delta 168.4,142.1,138.1,131.6,130.9,127.4,127.3,121.1,20.2$. One pair of carbons were coincident in the ${ }^{13} \mathrm{C}$ NMR. 
Representative Procedure for the Suzuki Cross-Coupling Reactions. $\mathrm{Pd}(\mathrm{OAc})_{2}(5.6 \mathrm{mg}$, $0.025 \mathrm{mmol})$, phosphine $(0.0625 \mathrm{mmol})$, boronic acid $(1.20 \mathrm{mmol})$ and $\mathrm{Na}_{2} \mathrm{CO}_{3}(0.21 \mathrm{~g}, 2.0$ mmol) were added to a round bottom equipped with a stir bar and rubber septum while in the dry box. Upon removing from dry box the arylbromide $(1.00 \mathrm{mmol})$ and degassed $1: 1 \mathrm{CH}_{3} \mathrm{CN}: \mathrm{H}_{2} \mathrm{O}$ $(10 \mathrm{~mL})$ were added via syringe. The reaction was placed in a pre-heated oil bath and allowed to stir until determined to be complete by GC (1-4 hours). The reaction was poured into saturated sodium carbonate $(50 \mathrm{ml})$, extracted with ethyl acetate $(3 \times 30 \mathrm{~mL})$, and dried with $\mathrm{MgSO}_{4}$. Products were purified by flash chromatography on silica gel.

4-Methylbiphenyl ${ }^{9}$ (entry 1). Using the general procedure, 4-bromotoluene (180.7 mg, 1.06 mmol) was coupled with phenylboronic acid (146 mg, $1.20 \mathrm{mmol})$ using TMAPTS/Pd(OAc) $)_{2}$ at $50{ }^{\circ} \mathrm{C}$. The crude product was chromatographed eluting with hexane: $\mathrm{CH}_{2} \mathrm{Cl}_{2}$ :EtOAc $(75: 20: 5)$ to give 4-methylbiphenyl as a colorless, low-melting point solid (169.2 mg, 95\% yield). ${ }^{1} \mathrm{HNMR}$ $\left(360 \mathrm{MHz}, \mathrm{CDCl}_{3}\right): \delta 7.56(\mathrm{~d}, J=1.23 \mathrm{~Hz}, 2 \mathrm{H}), 7.46(\mathrm{~d}, J=8.02 \mathrm{~Hz}, 2 \mathrm{H}), 7.39(\mathrm{~m}, 1 \mathrm{H}), 7.37(\mathrm{~m}$, $2 \mathrm{H}), 7.25(\mathrm{~d}, J=8.02,2 \mathrm{H}), 2.36(\mathrm{~s}, 3 \mathrm{H})$.

4-Methoxybiphenyl ${ }^{9}$ (entry 2$)$. Using the general procedure, 4-bromoanisole (188.2 mg, $1.01 \mathrm{mmol})$ was coupled with phenylboronic acid (146 mg, $1.20 \mathrm{mmol})$ using TXPTS/Pd(OAc) $)_{2}$ at $80{ }^{\circ} \mathrm{C}$. The crude product was chromatographed eluting with hexane: $\mathrm{CH}_{2} \mathrm{Cl}_{2}: \mathrm{EtOAc}(75: 20: 5)$ to give 4-methoxybiphenyl as a white solid (183.0 mg, $99 \%$ yield). mp 84-86 ${ }^{\circ} \mathrm{C}$ (lit. ${ }^{5} \mathrm{mp} 86-90$ $\left.{ }^{\circ} \mathrm{C}\right) .{ }^{1} \mathrm{HNMR}\left(360 \mathrm{MHz}, \mathrm{CDCl}_{3}\right): \delta 7.54(\mathrm{~m}, \mathrm{H}), 7.44(\mathrm{~m}, 2 \mathrm{H}), 7.29(\mathrm{~m}, 2 \mathrm{H}), 6.97(\mathrm{~d}, J=8.63 \mathrm{~Hz}$, $2 \mathrm{H}), 3.85$ (s, 3H).

4-Fluoro-4'-methylbiphenyl ${ }^{10}$ (entry 3 ). Using the general procedure, 4-bromotoluene (171.0mg, $1.00 \mathrm{mmol}$ ) was coupled with 4-fluorophenylboronic acid (169 $\mathrm{mg}, 1.20 \mathrm{mmol})$ using TXPTS $/ \mathrm{Pd}(\mathrm{OAc})_{2}$ at $50{ }^{\circ} \mathrm{C}$. The crude product was chromatographed eluting with hexane: $\mathrm{CH}_{2} \mathrm{Cl}_{2}$ :EtOAc (75:20:5) to give 4-methyl-4-fluorobiphenyl as a white solid (176.6 mg, 94\% yield). $\mathrm{mp} 74-77{ }^{\circ} \mathrm{C} .{ }^{1} \mathrm{HNMR}\left(360 \mathrm{MHz}, \mathrm{CDCl}_{3}\right): \delta 7.52(\mathrm{~d}, J=8.63 \mathrm{~Hz}, 2 \mathrm{H}), 7.43(\mathrm{~d}$, $J=8.02 \mathrm{~Hz}, 2 \mathrm{H}), 7.23$ (d, $J=8.02 \mathrm{~Hz}, 2 \mathrm{H}), 7.10$ (d, $J=8.64 \mathrm{~Hz}, 2 \mathrm{H}), 2.39$ (s, 3H).

4-Methoxy-4'-methylbiphenyl ${ }^{11}$ (entry 4). Using the general procedure, 4-bromotoluene (173.8 mg, $1.00 \mathrm{mmol}$ ) was coupled with 4-methoxyphenylboronic acid (182 $\mathrm{mg}, 1.20 \mathrm{mmol})$ using TXPTS/Pd(OAc) $)_{2}$ at $50{ }^{\circ} \mathrm{C}$. The crude product was chromatographed eluting with hexane: $\mathrm{CH}_{2} \mathrm{Cl}_{2}$ :EtOAc (75:20:5) to give 4-methoxy-4'-methylbiphenyl as a white solid (185.5 mg, 92\% yield). mp 106-108 ${ }^{\circ} \mathrm{C} .{ }^{1} \mathrm{HNMR}\left(360 \mathrm{MHz}, \mathrm{CDCl}_{3}\right): \delta 7.49(\mathrm{~d}, J=8.63 \mathrm{~Hz}, 2 \mathrm{H}), 7.44$ (d, $8.02 \mathrm{~Hz}, 2 \mathrm{H}), 7.22(\mathrm{~d}, J=8.02 \mathrm{~Hz}, 2 \mathrm{H}), 6.96(\mathrm{~d}, J=8.63 \mathrm{~Hz}, 2 \mathrm{H}), 3.83(\mathrm{~s}, 3 \mathrm{H}), 2.38$ (s, 3H). ${ }^{13} \mathrm{CNMR}\left(90.6 \mathrm{MHz}, \mathrm{CDCl}_{3}\right)$ : $\delta$ 159.6, 138.0, 136.3, 129.4, 127.9, 127.7, 126.6, 114.1, 55.3, 21.2.

4-Acetylbiphenyl ${ }^{12}$ (entry 5). Using the general procedure, 4'-bromoacetophenone (202.7 $\mathrm{mg}, 1.02 \mathrm{mmol}$ ) was coupled with phenylboronic acid (146 mg, $1.20 \mathrm{mmol}$ ) using TXPTS/Pd(OAc) $)_{2}$ at $50{ }^{\circ} \mathrm{C}$. The crude product was chromatographed eluting with hexane: $\mathrm{CH}_{2} \mathrm{Cl}_{2}$ :EtOAc (75:20:5) to give 4-acetylbiphenyl as a white solid (182.1 $\mathrm{mg}, 91 \%$ yield). mp 115-117 ${ }^{\circ} \mathrm{C}$ (lit mp 119-120 ${ }^{\circ} \mathrm{C}$ ). ${ }^{1} \mathrm{HNMR}\left(360 \mathrm{MHz}, \mathrm{CDCl}_{3}\right): \delta 8.03$ (d, J=8.63 Hz, $2 \mathrm{H}), 7.68(\mathrm{~d}, J=8.63 \mathrm{~Hz}, 2 \mathrm{H}), 7.62(\mathrm{~d}, J=7.39 \mathrm{~Hz}, 2 \mathrm{H}), 7.51(\mathrm{~m}, 3 \mathrm{H}), 2.69(\mathrm{~s}, 3 \mathrm{H}) .{ }^{13} \mathrm{CNMR}$ $\left(90.6 \mathrm{MHz}, \mathrm{CDCl}_{3}\right)$ : $\delta 197.7,145.8,139.9,135.8,128.9,128.8,128.2,127.3,127.2,26.6$. 
4-Acetyl-4'-methoxybiphenyl ${ }^{9}$ (entry 6). Using the general procedure, 4'bromoacetophenone $(210.3 \mathrm{mg}, 1.06 \mathrm{mmol}$ ) was coupled with 4-methoxyphenylboronic acid using TXPTS/Pd(OAc) $)_{2}$ at $50{ }^{\circ} \mathrm{C}$. The crude product was chromatographed eluting with hexane: $\mathrm{CH}_{2} \mathrm{Cl}_{2}$ :EtOAc (75:20:5) to give 4-acetyl-4'-methoxybiphenyl as a white solid (222.6 mg, 93\% yield). mp 149-151 ${ }^{\circ} \mathrm{C}\left(.{ }^{1} \mathrm{HNMR}\left(360 \mathrm{MHz}, \mathrm{CDCl}_{3}\right): \delta 8.03(\mathrm{~d}, J=8.63 \mathrm{~Hz}, 2 \mathrm{H}), 7.65\right.$ (d, $J=8.63 \mathrm{~Hz}, 2 \mathrm{H}), 7.58$ (d, $J=9.25 \mathrm{~Hz}, 2 \mathrm{H}), 6.99$ (d, J=8.63 Hz, 2H), 3.87 (s, 3H), 2.63 (s, 3H).

4-Fluoro-4'-methoxybiphenyl ${ }^{13}$ (entry 7). Using the general procedure, 4-bromoanisole (186.8 mg, $1.00 \mathrm{mmol}$ ) was coupled with 4-fluorophenylboronic acid (169 mg, $1.20 \mathrm{mmol}) \mathrm{using}$ TMAPTS $/ \mathrm{Pd}(\mathrm{OAc})_{2}$ at $50{ }^{\circ} \mathrm{C}$. The crude product was chromatographed eluting with hexane: $\mathrm{CH}_{2} \mathrm{Cl}_{2}$ :EtOAc (75:20:5) to give 4-fluoro-4'-methoxybiphenyl as a white solid (194.7 mg, 97\% yield). $\mathrm{mp} 86-88{ }^{\circ} \mathrm{C} .{ }^{1} \mathrm{HNMR}\left(360 \mathrm{MHz}, \mathrm{CDCl}_{3}\right): \delta 7.49(\mathrm{~m}, 4 \mathrm{H}), 7.12(\mathrm{~d}, J=8.63 \mathrm{~Hz}$, $2 \mathrm{H}), 6.96(\mathrm{~d}, J=6.78 \mathrm{~Hz}, 2 \mathrm{H}), 3.84(\mathrm{~s}, 3 \mathrm{H})$.

4-Methoxy-2-methylbiphenyl ${ }^{14}$ (entry 8 ). Using the general procedure, 4-bromo-3methylanisole (199.4 mg, $0.99 \mathrm{mmol})$ was coupled with phenylboronic acid (146 mg, 1.20 $\mathrm{mmol})$ using TXPTS/Pd(OAc) $)_{2}$ at $50{ }^{\circ} \mathrm{C}$. The crude product was chromatographed eluting with hexane: $\mathrm{CH}_{2} \mathrm{Cl}_{2}$ :EtOAc (75:20:5) to give 4-methoxy-2-methylbiphenyl (174.8 $\mathrm{mg}, 88 \%$ yield as a pale yellow oil). ${ }^{1} \mathrm{HNMR}\left(360 \mathrm{MHz}, \mathrm{CDCl}_{3}\right): \delta 7.30(\mathrm{~m}, 5 \mathrm{H}), 7.15(\mathrm{~d}, 1 \mathrm{H}, J=8.63 \mathrm{~Hz}), 6.69(\mathrm{~m}$, 2H), 3.67 (s, 3H), 2.15 (s, 3H). ${ }^{13} \mathrm{CNMR}\left(90.6 \mathrm{MHz} \mathrm{CDCl}_{3}\right): \delta 158.8,141.6,136.4,134.5$, $130.6,129.2,127.9,126.3,115.6,111.0,60.0,13.9$.

2,4'-Dimethylbiphenyl ${ }^{15}$ (entry 9). Using the general procedure, 4-bromotoluene (173.8 mg, $1.02 \mathrm{mmol}$ ) was coupled with 2-methylphenylboronic acid (163 mg, $1.20 \mathrm{mmol})$ using TMAPTS/Pd(OAc $)_{2}$ at $80{ }^{\circ} \mathrm{C}$. The crude product was chromatographed eluting with hexane: $\mathrm{CH}_{2} \mathrm{Cl}_{2}$ :EtOAc (75:20:5) to give 2,4'-dimethylbiphenyl as a pale yellow oil (146.6 mg, 86\% yield). ${ }^{1} \mathrm{HNMR}\left(360 \mathrm{MHz}, \mathrm{CDCl}_{3}\right): \delta 7.07(\mathrm{~m}, 8 \mathrm{H}), 2.23(\mathrm{~s}, 3 \mathrm{H}), 2.13(\mathrm{~s}, 3 \mathrm{H}) .{ }^{13} \mathrm{C} \mathrm{NMR}$ $\left(90.6 \mathrm{MHz} \mathrm{CDCl}_{3}\right): \delta 142.1,139.4,136.6,135.6,130.6,130.2,129.4,129.1,127.4,126.1,21.4$, 20.8 .

4-Methoxy-2,2'-dimethylbiphenyl (entry 10). Using the general procedure, 4-bromo-3methylanisole $(199.4 \mathrm{mg}, 0.99 \mathrm{mmol})$ was coupled with 2-methylphenylboronic acid (163 $\mathrm{mg}$, $1.20 \mathrm{mmol})$ using TXPTS/Pd(OAc $)_{2}$ at $50{ }^{\circ} \mathrm{C}$. The crude product was chromatographed eluting with hexane: $\mathrm{CH}_{2} \mathrm{Cl}_{2}$ :EtOAc $(75: 20: 5)$ to give 4-methoxy-2, 2'-dimethylbiphenyl as a pale yellow oil (157.0 mg, 75\% yield). ${ }^{1} \mathrm{HNMR}\left(360 \mathrm{MHz}, \mathrm{CDCl}_{3}\right): \delta 7.13(\mathrm{~m}, 3 \mathrm{H}), 6.98(\mathrm{~d}, J=7.4 \mathrm{~Hz}$, $1 \mathrm{H}), 6.88(\mathrm{~d}, J=8.63 \mathrm{~Hz}, 1 \mathrm{H}), 6.69(\mathrm{~m}, 2 \mathrm{H}), 3.69(\mathrm{~s}, 3 \mathrm{H}), 2.02(\mathrm{~s}, 3 \mathrm{H}), 1.85(\mathrm{~s}, 3 \mathrm{H}) ;{ }^{13} \mathrm{C} \mathrm{NMR}$ $\left(90.6 \mathrm{MHz}_{\mathrm{CDCl}}\right): \delta$ 158.6, 141.2, 137.1, 136.2, 134.1, 130.2, 129.8, 129.7, 127.0, 125.4, $115.2,110.8,55.0,20.0,19.8$.

2,6-Dimethylbiphenyl ${ }^{16}$ (entry 11). Using the general procedure, 2-bromo- $m$-xylene (187.5 $\mathrm{mg}, 1.01 \mathrm{mmol})$ was coupled with phenylboronic $(146 \mathrm{mg}, 1.20 \mathrm{mmol})$ acid using TXPTS $/ \mathrm{Pd}(\mathrm{OAc})_{2}$ at $50{ }^{\circ} \mathrm{C}$. The crude product was chromatographed eluting with hexane: $\mathrm{CH}_{2} \mathrm{Cl}_{2}$ :EtOAc (75:20:5) to give 2,6-dimethylbiphenyl as a pale yellow oil (145.3 mg, $78 \%$ yield). ${ }^{1} \mathrm{HNMR}\left(360 \mathrm{MHz}, \mathrm{CDCl}_{3}\right): \delta 7.71(\mathrm{~m}, 2 \mathrm{H}), 7.62(\mathrm{~m}, 1 \mathrm{H}), 7.43(\mathrm{~m}, 5 \mathrm{H}), 2.36(\mathrm{~s}$, $6 \mathrm{H})$. 
4-Fluoro-2',6'-dimethylbiphenyl (entry 12). Using the general procedure, 2-bromo- $m$ xylene $(187.5 \mathrm{mg}, 1.01 \mathrm{mmol})$ was coupled with 4-fluorophenylboronic acid $(169 \mathrm{mg}, 1.20$ mmol) using TMAPTS/Pd(OAc $)_{2}$ at $50{ }^{\circ} \mathrm{C}$. The crude product was chromatographed eluting with hexane: $\mathrm{CH}_{2} \mathrm{Cl}_{2}$ :EtOAc (75:20:5) to give 4-fluoro-2',6'-dimethylbiphenyl (175.8 mg, 87\% yield as a pale yellow oil). ${ }^{1} \mathrm{HNMR}\left(360 \mathrm{MHz}, \mathrm{CDCl}_{3}\right): \delta 7.12(\mathrm{~m}, 7 \mathrm{H}), 2.05(\mathrm{~s}, 6 \mathrm{H}) .{ }^{13} \mathrm{C}$ NMR $\left(90.6 \mathrm{MHz}, \mathrm{CDCl}_{3}\right): \delta 161.8\left(\mathrm{~d},{ }^{I} J_{\mathrm{C}-\mathrm{F}}=244.14 \mathrm{~Hz}\right), 140.8,136.9\left(\mathrm{~d}, J_{\mathrm{C}-\mathrm{F}}=4.16 \mathrm{~Hz}\right), 136.2,130.6$ $\left(\mathrm{d}, J_{\mathrm{C}-\mathrm{F}}=6.93 \mathrm{~Hz}\right), 127.3,127.2115 .5\left(\mathrm{~d}, J_{\mathrm{C}}=22.20 \mathrm{~Hz}\right), 20.8$.

(1) Gulyás, H.; Szollöy, Á.; Hanson, B. E.; Bakos, J. Tetrahedron Lett. 2002, 43, 2543-2546.

(2) Fujiwara, Y.; Moritani, I.; Danno, S.; Asano, R.; Teranishi, S. Journal of the American Chemical Society 1969, 91, 7166-7169.

(3) Katrizky, A. R.; Tymoshenko, D. O.; Belyakov, S. A. Journal of Organic Chemistry 1999, 64, 3332-3334.

(4) Selvakumar, K.; Zapf, A.; Beller, M. Org. Lett. 2002, 4, 3031-3033.

(5) Cleland, G. H. Journal of Organic Chemistry 1969, 34, 744-747.

(6) Saito, T.; Jayamizu, K.; Ymamgisawa, M.; Yamamoto, O.; Wasada, N.; Someno, K.; Kinugasa, S.; Tanabe, K.; Tamura, T.; Hiraishi, J., Integrated Spectral Data Base System for Organic Compounds, http://www.aist.go.jp/RIODB/SDBS/menu-e.html, 2003

(7) Yu, J.; Spencer, J. B. Journal of Organic Chemistry 1997, 62, 8618-8619.

(8) Liu, J.-M.; Young, J.-J.; Li, Y.-J.; Sha, C.-K. Journal of Organic Chemistry 1986, $51,1120-1123$.

(9) Shaughnessy, K. H.; Booth, R. S. Org. Lett. 2001, 3, 2757-2759.

(10) Nishimura, M.; Ueda, M.; Miyaura, N. Tetrahedron 2002, 58, 5779-5787.

(11) Roy, A. H.; Hartwig, J. F. Journal of the American Chemical Society 2003, 125, 8704-8705.

(12) Zhu, L.; Duquette, J.; Zhang, M. Journal of Organic Chemistry 2003, 68, 37293732 . 13086

(13) Ueda, M.; Saitoh, A.; Oh-Tani, S.; Miyaura, N. Tetrahedron 1998, 54, 13079-

(14) Marx, J. N.; Argyle, J. C.; Norman, L. R. Journal of the American Chemical Society 1974, 96, 2121-2129.

(15) Littke, A. F.; Dai, C.; Fu, G. C. J. Am. Chem. Soc. 2000, 122, 4020-4028.

(16) Littke, A. F.; Schwarz, L.; Fu, G. C. Journal of the American Chemical Society 2003, 124, 6343-6348. 